\title{
Grape seed proanthocyanidin inhibits inflammatory responses in hepatic stellate cells by modulating the MAPK, Akt and NF-кB signaling pathways
}

\author{
JIN-WOO LEE ${ }^{1,2}$, YOUNG IL KIM ${ }^{2}$, YOUNGCHUL KIM ${ }^{3}$, MINJI CHOI ${ }^{4}$, SEOYEON MIN ${ }^{5}$, \\ YONG HOON JOO ${ }^{1}$, SUNG-VIN YIM ${ }^{6}$ and NAMHYUN CHUNG ${ }^{1}$
}

\author{
${ }^{1}$ Department of Biosystems and Biotechnology, College of Life Sciences and Biotechnology, \\ Korea University, Seoul 02841; ${ }^{2}$ Medical Science Research Institute, Kyung Hee University Medical Center; \\ Departments of ${ }^{3}$ Internal Medicine and ${ }^{4}$ Science in Korean Medicine, College of Korean Medicine, Kyung Hee University; \\ ${ }^{5}$ Department of Nephrology, College of Medicine, Kyung Hee University; ${ }^{6}$ Department of Clinical Pharmacology, \\ College of Medical Science, Kyung Hee University, Seoul 02447, Republic of Korea
}

Received October 21, 2016; Accepted May 10, 2017

DOI: $10.3892 /$ ijmm.2017.2997

\begin{abstract}
In the present study, we aimed to investigate the molecular mechanisms and prophylactic effects of grape seed proanthocyanidin (GSP) on lipopolysaccharide (LPS)-stimulated human hepatic stellate cells (HSCs). Cell counting and MTT assays were used to assess cell viability in the absence or presence of GSP. Reverse transcription-quantitative PCR (RT-qPCR) was performed for several inflammation-related genes (NOD1, NOD2, TLR2, TLR4, IL-1 $\beta$, IL-6, IL-8, iNOS and COX-2). The expression of anti-inflammatory cell signaling molecules, including c-Jun N-terminal kinase (JNK), p38, extracellular signal regulated kinase (ERK), Akt, nuclear factor- $\kappa \mathrm{B}(\mathrm{NF}-\mathrm{\kappa B})$, inhibitory- $\kappa \mathrm{B} \alpha(\mathrm{I} \kappa \mathrm{B} \alpha)$, iNOS and COX-2, was evaluated by western blot analysis. Finally, IL-8 levels in the culture supernatant of HSCs were measured by ELISA. Pretreatment with GSP before LPS treatment significantly suppressed the mRNA expression of pro-inflammatory cytokines such as IL-1 $\beta$, IL-6 and IL-8. GSP inhibited mRNA expression of LPS-induced TLR4, NOD2 and COX-2, in addition to inhibiting the expression of iNOS. GSP also inhibited LPS-induced NF- $\mathrm{kB}$ activation and I $\mathrm{B} \alpha$ phosphorylation. Concomitantly, GSP dose-dependently suppressed the activation of MAP kinases (JNK, ERK and p38)
\end{abstract}

Correspondence to: Professor Namhyun Chung, Department of Biosystems and Biotechnology, College of Life Sciences and Biotechnology, Korea University, 145 Anam-ro, Seongbuk-gu, Seoul 02841, Republic of Korea

E-mail: nchung@korea.ac.kr

Professor Sung-Vin Yim, Department of Clinical Pharmacology, College of Medical Science, Kyung Hee University, 26 Kyung Hee Dae-ro, Dongdaemun-gu, Seoul 02447, Republic of Korea E-mail: ysvin@khu.ac.kr

Key words: cyclooxygenase-2, grape seed proanthocyanidin, hepatic stellate cells, inducible nitric oxide synthase, mitogen-activated protein kinase, Toll-like receptors and Akt in LPS-stimulated HSCs. These data suggest that GSP inhibits inflammatory responses in HSCs by inactivating the $\mathrm{NF}-\kappa \mathrm{B}$ signaling pathway via MAP kinases. Thus, GSP may be considered as a novel drug for the treatment of hepatic inflammation, infectious diseases and fibrosis.

\section{Introduction}

Grape seed proanthocyanidin (GSP), referred to as condensed tannins, includes a high content of flavonoids. The free radical-scavenging abilities of GSP were found to reduce the risk of cancer (1), blood clotting (2) and cardiovascular disease (3). Proanthocyanidins are compounds naturally found in vegetables, bark, fruits and seeds; grape seeds are a particularly rich source of proanthocyanidins in both quantity and variety (4). A variety of proanthocyanidins have multiple functions such as antibacterial, antiviral $(5)$, anticarcinogenic $(5,6)$ and anti-inflammatory activity (7). GSP is sold in the market as a dietary supplement because of its potential antioxidant activity, together with its low toxicity and lack of genotoxic potential (8). However, there have been no studies on the anti-inflammatory effects of GSP in human hepatic stellate cells (HSCs).

Chronic liver disease is often caused by inflammation. In all disease stages, inflammation is observed and is characterized by the development of hepatocellular carcinoma, cirrhosis and fibrosis, which are mainly of viral or autoimmune origin, or are caused by alcohol abuse (9). HSCs are the major players in liver inflammation and fibrogenesis. For example, HSC activation and the subsequent matrix secretion by activated HSCs induce liver fibrosis, leading to cirrhosis in chronic liver injury (10). The reciprocal relationship between HSCs and precancerous hepatocytes or hepatoma cells promotes tumorigenesis, migration and invasion of cancer cells, and formation of metastasis (11). Specifically, both activated and proliferating HSCs play key roles in the inflammation-fibrosis-carcinoma axis, whereas apoptotic HSCs promote fibrosis resolution (11). The human HSC cell line, LX-2, exhibits the typical charac- 
teristics of HSCs under primary culture. Thus, LX-2 cells are considered a novel tool for analyzing hepatic fibrosis (12).

After liver injury, recruited inflammatory cells accumulate in the damaged site. A wide repertoire of pro-inflammatory and anti-inflammatory compounds including chemokines, cytokines and growth factors mediate the inflammatory response of immune cells during the process of fibrosis (13). HSCs also play an active role in the progression of inflammation by interacting with various immune cells (14). Almost all inflammatory stimuli converge on HSCs. As inflammation is an important factor in the pathogenesis of liver fibrosis, managing inflammatory responses is an important strategy for treating hepatic fibrosis (15).

Medicinal plants produce compounds that suppress inflammation, suggesting that their extracts could be used for the treatment of symptoms of fibrosis. For example, these extracts reduce liver fibrosis by decreasing hepatic secretion of inflammatory cytokines at the protein and mRNA levels in the liver (16). Specifically, vegetal compounds target pro-inflammatory cytokines and chemokines such as interleukin-1 $\beta$ (IL-1 $\beta$ ), IL-2, IL-6, IL-8, interferon- $\gamma$ (IFN- $\gamma$ ) and tumor necrosis factor- $\alpha$ (TNF- $\alpha)$ (17). Moreover, liver inflammation is suppressed by upregulation of the hepatic levels of anti-inflammatory cytokines (IL-4, IL-10 and IL-13), together with inhibition of the expression of inducible nitric oxide synthase (iNOS) and cyclooxygenase-2 (COX-2) (16). Toll-like receptors (TLRs) 2 and 4 are central mediators of inflammation during liver fibrosis. TLR ligands consist of pathogen-associated molecular patterns (PAMPs) and damageassociated molecular patterns (DAMPs) (18). The high mobility group box 1 (HMGB1)-TLR2/TLR4-NF- $\kappa$ B signaling pathway is a potential therapeutic target for suppression of inflammation in liver fibrosis. In the present study, we investigated the underlying molecular mechanisms and prophylactic effects of GSP on lipopolysaccharide (LPS)-stimulated LX-2 cells.

\section{Materials and methods}

Materials. GSP from Vitis vinifera was kindly supplied by Hanlim Pharmaceutical (Seoul, Korea). GSP contains proanthocyanidins as a major component $(80 \%)$, as well as several catechin monomers (19). GSP was solubilized in phosphate-buffered saline (PBS). Dulbecco's modified Eagle's medium (DMEM) and other related products were purchased from Gibco (Grand Island, NY, USA). Fetal bovine serum (FBS) was obtained from PAA Laboratories (Linz, Austria). Sigma-Aldrich (St. Louis, MO, USA) was the supplier of all other chemicals of analytical grade. R\&D Systems (Minneapolis, MN, USA) was the supplier of the antibody against iNOS (MAB9502) and the enzymelinked immunosorbent assay (ELISA) kit for IL-8 (D8000C). Antibodies against COX-2 (\#4842) and $\beta$-actin (\#4970) as well as horseradish peroxidase-conjugated anti-mouse (\#7076) and anti-rabbit (\#7074) IgG were purchased from Cell Signaling Technology (Beverly, MA, USA). Anti-phospho or total antibodies to JNK (\#9258, \#9251 for total and phospho form), Akt (\#4691, \#4060 for total and phospho form), ERK (\#4695, \#4370 for total and phospho form), p38 (\#8690,\#4511 for total and phospho form), I $\kappa \mathrm{B} \alpha$ (\#9242, \#9246 for total and phospho form) and NF- $\kappa$ B (\#8242,\#3031 for total and phospho form) were also purchased from Cell Signaling Technology.
Cell culture. The LX-2 cell line is an immortalized human HSC line, and was provided by Dr Scott L. Friedman (Mount Sinai Hospital, New York, NY, USA). LX-2 cells were grown as previously described (12). They were grown in monolayers with DMEM supplemented with $2 \%(\mathrm{v} / \mathrm{v})$ heat-inactivated FBS, $100 \mu \mathrm{g} / \mathrm{ml}$ streptomycin and $100 \mathrm{U} / \mathrm{ml}$ penicillin at $37^{\circ} \mathrm{C}$ in a humidified atmosphere of $5 \% \mathrm{CO}_{2}$ in air. Then, after rinsing with PBS, they were starved by incubation in a serumfree medium for $24 \mathrm{~h}$. The treated LX-2 cells were exposed to LPS $(1 \mu \mathrm{g} / \mathrm{ml})$ with or without GSP.

Cell viability. The anti-proliferative effects of GSP on LX-2 cells were determined as follows. Cells were grown on 6-well plates (1x $10^{5} /$ well) for 24 or $48 \mathrm{~h}$, after which the indicated concentration of GSP was added; controls received $0.01 \%$ PBS. After the indicated incubation times, the cells in each well were harvested with trypsin-EDTA solution (JBI, Seoul, Korea) and washed once with PBS containing 5\% FBS. Then, the number of cells was counted using an ADAM-MC cell counter (NanoEnTeK, Seoul, Korea), and the effects of GSP on cell proliferation were examined by the 3-(4,5-dimethylthiazol-2-yl)-2,5-diphenyltetrazolium bromide (MTT) assay (20). A total of $1.5 \times 10^{4} \mathrm{LX}-2$ cells/well were plated in 96-well culture plates and incubated for $24 \mathrm{~h}$. Then, GSP concentrations of $1-100 \mu \mathrm{g} / \mathrm{ml}$ were added to the cell culture; cells were incubated for an additional 24 and $48 \mathrm{~h}$. At predetermined times following treatment with GSP, the medium was replaced with MTT $(20 \mu 1,5 \mathrm{mg} / \mathrm{ml})$ in each well. Incubation was continued at $37^{\circ} \mathrm{C}$ for an additional $2 \mathrm{~h}$, after which the plate contents were centrifuged and the supernatants were disposed of. Formazan precipitates were dissolved with $200 \mu$ l dimethyl sulphoxide. The absorbance was obtained at $570 \mathrm{~nm}$ in comparison to $650 \mathrm{~nm}$ as a blank using an E-max microplate reader (Molecular Devices, Sunnyvale, CA, USA). The change in percentage of cell proliferation in each well was expressed in comparison to the non-GSP-treated controls. All experiments were repeated three times independently.

RNA isolation, cDNA synthesis and reverse transcriptionquantitative PCR (RT-qPCR). Total RNA from the cells was obtained with TRIzol reagent (Invitrogen, Carlsbad, CA, USA). First-strand cDNA was synthesized by reverse transcription in a $20-\mu 1$ reaction mixture containing $1 \mathrm{mM}$ dNTPs, $1 \mu \mathrm{g}$ RNA, $1 \mathrm{X}$ reaction buffer, $5 \mu \mathrm{M}$ random primers, and 20 units of AMV reverse transcriptase (Promega, Madison, WI, USA). The individual sequences for the gene-specific primers used were as follows: IL-6, 5'-GTCTTGCCTGCT GCCTTC-3' and 5'-AGTGCCTCTTTGCTGCTTTC-3' (194 bp); IL-8, 5'-GACATACTCCAAACCTTTCCAC-3' and 5'-CTTCTCCACAAACCTCTGC-3' (160 bp); IL-1 $\beta$, 5'-TGA TGGCTTATTACAGTGGCAATG-3' and 5'-GTAG TGGTGGTCGGAGATTCG-3' (140 bp); TLR-2, 5'-TCTCCC ATTTCCGTCTTTTT-3' and 5'-GGTCTTGGTGTTCATT ATCTTC-3' (125 bp); TLR-4, 5'-GAAGCTGGTGGCTGT GGA-3' and 5'-TGATGTAGAACCCGCAAG-3' (213 bp); $\beta$-actin, 5'-GCGAGAAGATGACCCAGATC-3' and 5'-GGAT AGCACAGCCTGGATAG-3' (77 bp); NOD1, 5'-GTCACT GAGGTCCATCTGAAC-3' and 5'-CATCCACTCCTGG AAGAACCT-3' (363 bp); NOD2, 5'-CATGTGCTGCTAC GTGTTCTC-3' and 5'-CCTGCCACAATTGAAGAGGTG-3' (226 bp); iNOS, 5'-TGGATGCAACCCCATTGTC-3' and 

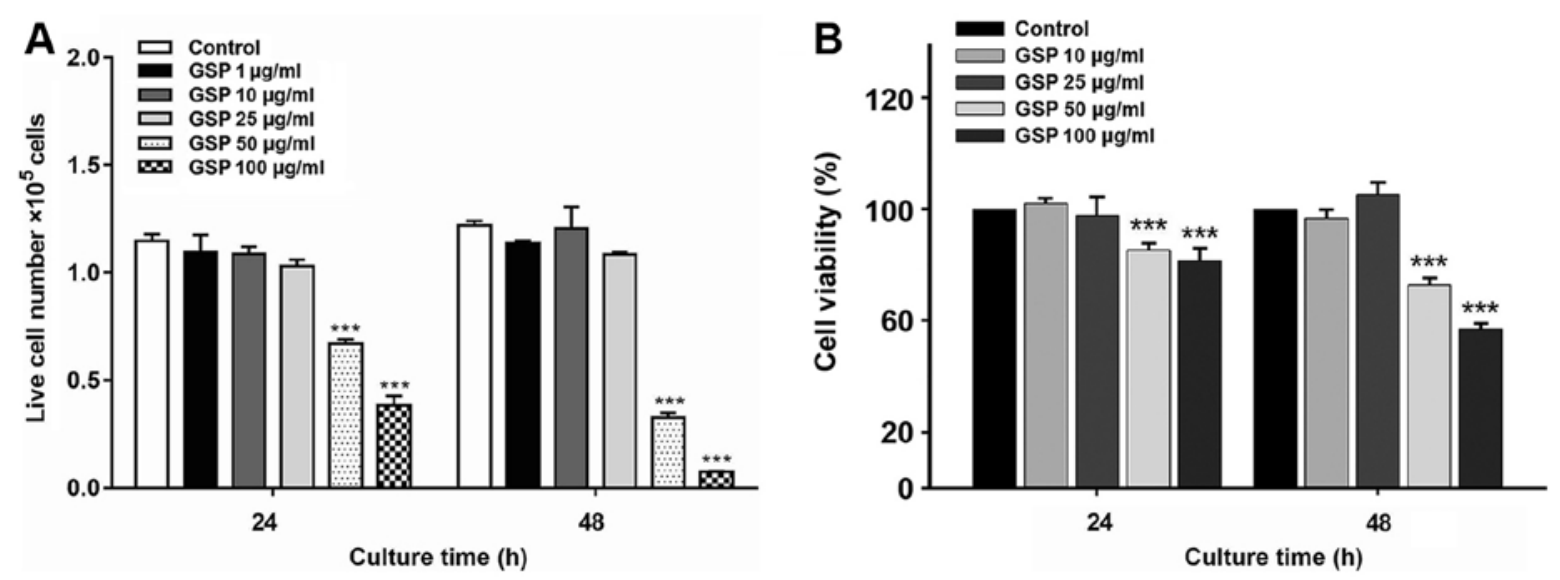

Figure 1. Effects of grape seed proanthocyanidin (GSP) on the cell viability of LX-2 cells. Cells were treated with the indicated concentrations of GSP for 24 and $48 \mathrm{~h}$. Cell numbers were determined using an automatic cell counter (A) and cell viability was determined by the MTT assay (B). Values are expressed as the mean \pm SD of three independent experiments. ${ }^{* * *}$ p $<0.001$ compared to lipopolysaccharide (LPS) alone.

5'-CCCGCTGCCCCAGTTT-3' (59 bp); COX-2, 5'-CAAAT CCTTGCTGTTCCCACCCAT-3' and 5'-GTGCACTGTGTT TGGAGTGGGTTT-3' (173 bp). Quantitative PCR (qPCR) was carried out using a StepOnePlus real-time PCR system (Applied Biosystems, Foster City, CA, USA). PCR was carried out with $1 \mu 1 \mathrm{cDNA}$ in $20 \mu \mathrm{l}$ reaction mixtures consisting of $1 \mu 1$ primers, $10 \mu \mathrm{l}$ Power SYBR-Green PCR Master Mix and $7 \mu \mathrm{l}$ PCR-grade water. The amplification protocols included an initial denaturation step $\left(95^{\circ} \mathrm{C}, 10 \mathrm{~min}\right), 40$ subsequent cycles of denaturation $\left(95^{\circ} \mathrm{C}, 15 \mathrm{sec}\right)$, and an annealing step $\left(60^{\circ} \mathrm{C}\right.$, $1 \mathrm{~min})$. The crossing point value $(\triangle \mathrm{CT})$ of each cDNA was applied to the formula $2^{\text {-(target gene - } \beta \text {-actin) }}$ to quantify the relative amounts of each cDNA.

Western blot analysis. Treated cells were washed with cold PBS to be lysed with lysis buffer (Cell Signaling Technology). Bicinchoninic acid (BCA) protein assay was employed to determine the total protein concentration, in accordance with the manufacturer's instructions. Protein $(30 \mu \mathrm{g})$ was mixed with loading buffer, boiled for $5 \mathrm{~min}$, and loaded onto $8-12 \%$ polyacrylamide gels. After electrophoresis, the proteins were transferred to PVDF membranes. After blocking the membranes with 5\% non-fat dried milk for $1 \mathrm{~h}$, they were incubated for $1 \mathrm{~h}$ in a solution of Tris-buffered saline with $0.05 \%$ Tween-20 (TBS-T) containing primary antibody at a dilution rate of 1:500-1:1,000. After washing with TBS-T, the membranes were soaked in TBS-T solution containing horseradish peroxidase-conjugated secondary antibodies at a dilution of 1:2,500 for $1 \mathrm{~h}$. After a second wash with TBS-T, target protein bands were visualized using an Enhanced Chemiluminescence kit (Thermo Scientific, Rockford, IL, USA). The target protein expression was visualized using a Davinch-Chemi Chemiluminescence Imaging system (Davinch-K Co., Seoul, Korea).

Measurement of IL-8. LX-2 cells were seeded in 6-well plates at a density of $6 \times 10^{5}$ cells/well and cultured with various doses of GSP for $4 \mathrm{~h}$ at $37^{\circ} \mathrm{C}$ before challenge with LPS $(1 \mu \mathrm{g} / \mathrm{ml})$ for $24 \mathrm{~h}$. After treatment, culture media were collected and subjected to centrifugation at 1,000 x g for $15 \mathrm{~min}$. The supernatants were analyzed with IL-8 ELISA kits (R\&D Systems).
Statistical analysis. All values are expressed as the mean \pm standard deviation (SD). SigmaPlot version 10 software (Systat Software Inc., Chicago, IL, USA) was used for statistical analyses. Student's t-test or one-way ANOVA was used for the determination of statistical significance of differences between the LPS-treated and GSP plus LPS-treated cells. In all analyses, a p-value of $<0.05$ was considered statistically significant.

\section{Results}

Effects of GSP on the viability of LPS-induced human HSCs. The cytotoxic effects of GSP on LX-2 cells were evaluated by exposing the cells to varying concentrations of GSP for 24 and $48 \mathrm{~h}$. The data are expressed as the number of cells (Fig. 1A) and the percentage of cell viability (Fig. 1B) compared to the controls. GSP did not exhibit cytotoxicity towards LX-2 cells at doses of 10 and $25 \mu \mathrm{g} / \mathrm{ml}$, which were the doses used for treatment with GSP in subsequent experiments.

Effects of GSP on the mRNA levels of NOD1, NOD2, TLR2 and TLR4 in LPS-stimulated LX-2 cells. The effects of GSP on the mRNA expression of NOD1, NOD2, TLR2 and TLR4 were investigated by qPCR. TLR2 and TLR4 are central intermediaries of inflammation in liver fibrosis. In particular, TLR4 may be involved in the increase in inflammation and fibrosis of the liver (21). In this study, LX-2 cells were pre-incubated with GSP before LPS treatment. LPS treatment caused significant increases in mRNA levels of TLR4 (Fig. 2B) and NOD2 (Fig. 2D) compared to the controls. The mRNA levels of NOD2 and TLR4 were decreased in the cells pretreated with GSP. In contrast, mRNA levels of TLR 2 and NOD1 were not significantly affected by LPS stimulation (Fig. 2A and C). Decreases in mRNA levels following GSP pretreatment were likely only due to the drug, and were independent of LPS stimulation.

Effects of GSP on mRNA levels of IL-1 $\beta, I L-6$ and IL-8 in $L P S$-stimulated $L X-2$ cells. We performed qPCR to evaluate the mRNA expression levels of pro-inflammatory cytokines including IL-1 $\beta$, IL-6 and IL- 8 in LPS-stimulated HSCs. As shown in Fig. 3, significant increases in cytokine expression were induced by LPS treatment; however, pretreatment with 
A

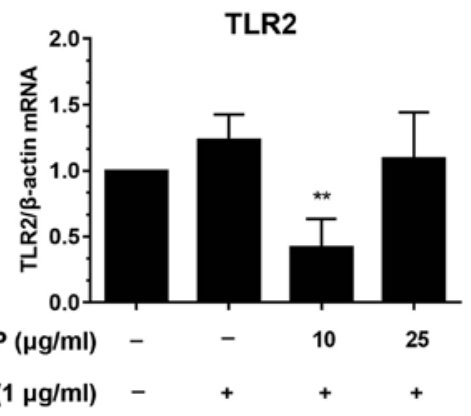

B

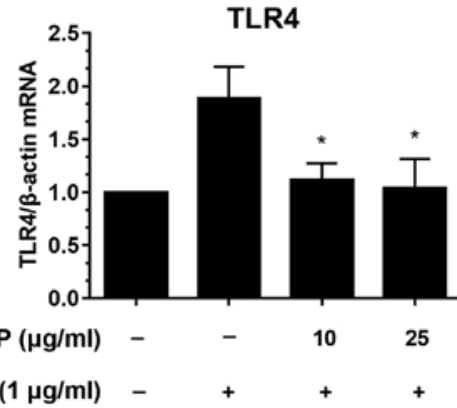

C

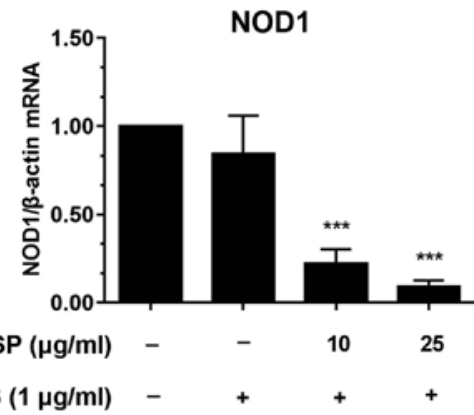

D

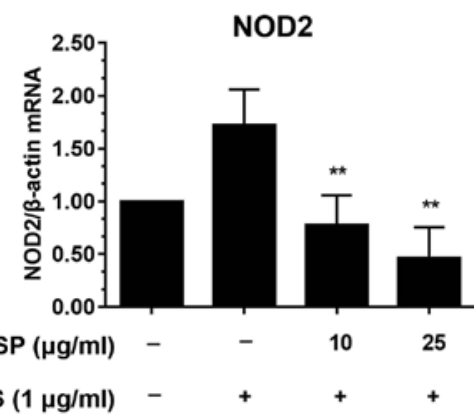

Figure 2. Effects of grape seed proanthocyanidin (GSP) on mRNA levels of NOD1, NOD2, TLR2 and TLR4 in lipopolysaccharide (LPS)-stimulated LX-2 cells. After cells were pretreated with GSP (10 and $25 \mu \mathrm{g} / \mathrm{ml})$ for $24 \mathrm{~h}$ and stimulated with LPS $(1 \mu \mathrm{g} / \mathrm{ml})$ for an additional $4 \mathrm{~h}$, and real-time quantitative PCR was performed to analyze mRNA levels. The crossing points of TLR2 (A), TLR4 (B), NOD1 (C) and NOD2 (D) with $\beta$-actin were entered into the formula: $2^{\text {-(target gene }-\beta \text {-actin) }}$. Data are expressed as the mean \pm SD of three independent experiments. ${ }^{*} \mathrm{p}<0.05,{ }^{* *} \mathrm{p}<0.01$ and ${ }^{* * * *} \mathrm{p}<0.001$ compared to LPS alone. TLR, Toll-like receptor; NOD1 and NOD2, nucleotide binding oligomerization domain containing 1 and 2 .

GSP decreased the mRNA expression levels. These findings indicated that GSP regulates immune responses by reducing the mRNA expression levels of pro-inflammatory genes.
A

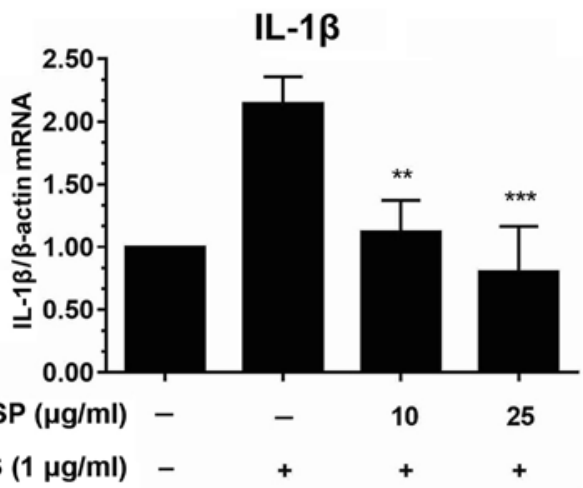

B

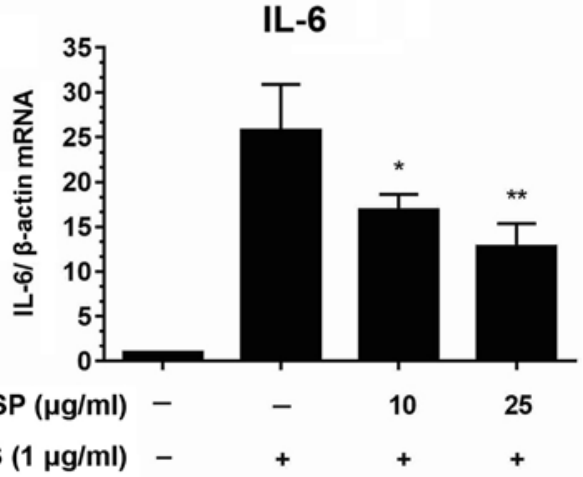

C IL-8

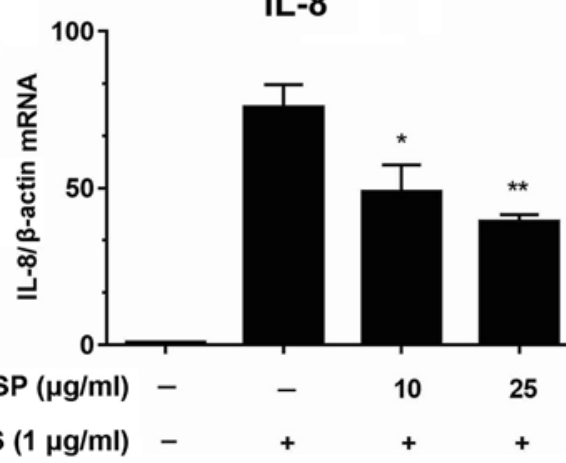

Figure 3. Effects of grape seed proanthocyanidin (GSP) on mRNA levels of interleukin-1 $\beta$ (IL-1 $\beta$ ), IL-6 and IL-8 in lipopolysaccharide (LPS)-stimulated LX-2 cells. Cells were pretreated with GSP (10 and $25 \mu \mathrm{g} / \mathrm{ml})$ for $24 \mathrm{~h}$ before stimulation with LPS $(1 \mu \mathrm{g} / \mathrm{ml})$ for $4 \mathrm{~h}$. The mRNA levels were measured by real-time quantitative PCR. The crossing points of IL-1 $\beta$ (A), IL-6 (B) and IL-8 (C) with $\beta$-actin were entered into the formula $2^{\text {-(target gene - } \beta \text {-actin) }}$. Data are expressed as the mean \pm SD of three independent experiments. ${ }^{*} \mathrm{p}<0.05$, ${ }^{* *} \mathrm{p}<0.01$ and ${ }^{* * *} \mathrm{p}<0.001$ compared to LPS alone.

Effects of GSP on $m R N A$ and protein expression of COX-2 and iNOS in LPS-induced LX-2 cells. Next, we performed qPCR and western blot analyses to investigate the effects of GSP on the expression levels of COX-2 and iNOS. Fig. 4A and B show that LPS treatment noticeably increased the mRNA expression levels of COX-2 and iNOS. In contrast, GSP pretreatment decreased the mRNA and protein levels of COX-2 and iNOS in the LPS-induced LX-2 cells (Fig. 4C and D).

Effects of GSP on phosphorylation of NF- $\kappa B$ in LPS-induced $L X-2$ cells. All major pro-inflammatory mediators are 

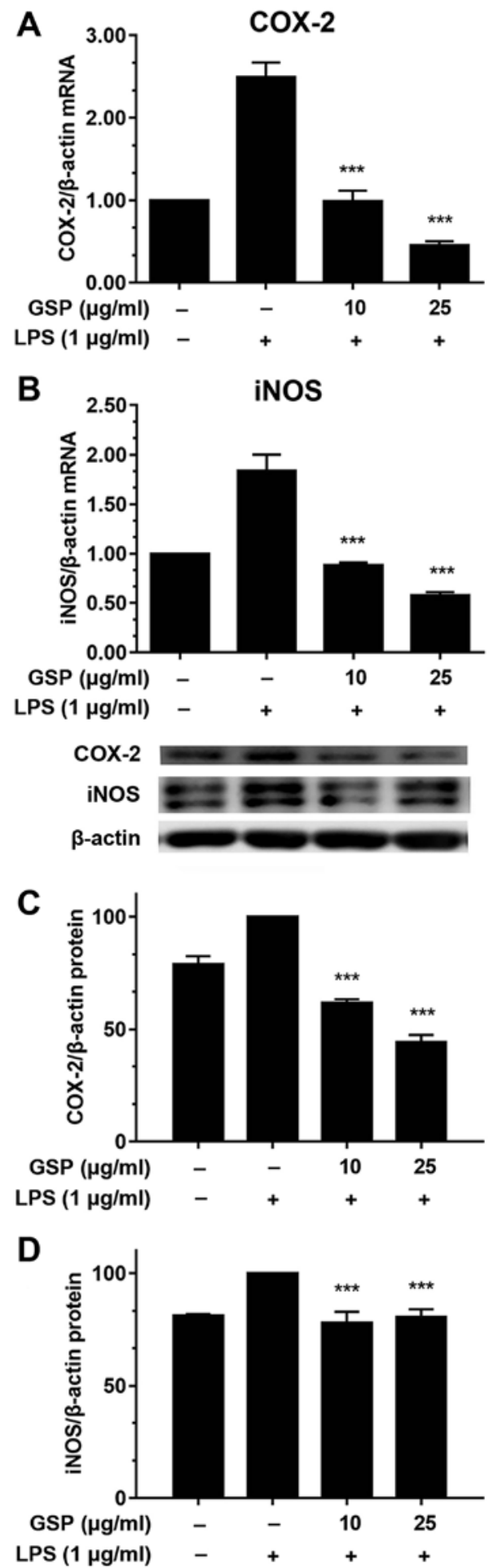

Figure 4. Effects of grape seed proanthocyanidin (GSP) on mRNA and protein expression of cyclooxygenase-2 (COX-2) and inducible nitric oxide synthase (iNOS) in lipopolysaccharide (LPS)-induced LX-2 cells. Cells were pretreated with different concentrations of GSP (10 and $25 \mu \mathrm{g} / \mathrm{ml}$ ) for $24 \mathrm{~h}$ followed by LPS stimulation for $4 \mathrm{~h}$. Levels of COX-2 (A) and iNOS mRNA (B) were measured by real-time quantitative PCR. The crossing points of COX-2 (A) and iNOS (B) with $\beta$-actin were entered into the formula $2^{\text {-(target gene - } \beta \text {-actin) }}$. Protein levels of COX-2 (C) and iNOS (D) were determined by western blot analyses. Data are expressed as the mean \pm SD of three independent experiments. ${ }^{* * *} \mathrm{p}<0.001$ compared to LPS alone.

induced via activation of $\mathrm{NF}-\kappa \mathrm{B}$ (22). Therefore, we hypothesized that the aforementioned findings were related

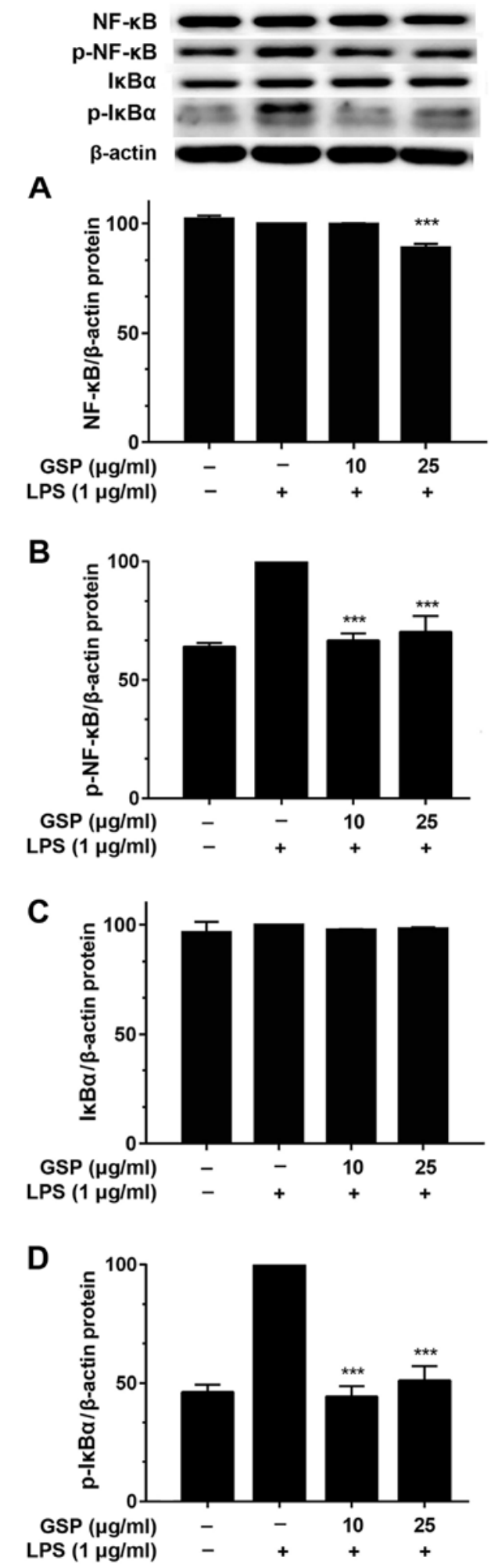

Figure 5. Effects of grape seed proanthocyanidin (GSP) on nuclear factor- $\kappa \mathrm{B}(\mathrm{NF}-\kappa \mathrm{B})$ p 65 phosphorylation and I $\mathrm{B} \alpha$ phosphorylation in lipopolysaccharide (LPS)-induced LX-2 cells. Cells were pretreated with GSP (10 and $25 \mu \mathrm{g} / \mathrm{ml}$ ) for $24 \mathrm{~h}$ and stimulated with LPS for a further $2 \mathrm{~h}$. Cytosolic proteins were prepared and analyzed by western blot analysis. The ratios of

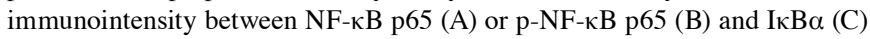
or $\mathrm{p}-\mathrm{I \kappa} \mathrm{B} \alpha(\mathrm{D})$ to $\beta$-actin were calculated. Data are expressed as the mean $\pm \mathrm{SD}$ of three independent experiments. ${ }^{* * *} \mathrm{p}<0.001$ compared to LPS alone.

to the NF- $\mathrm{B}$ signaling pathway. Thus, the effects of GSP on $\mathrm{I} \kappa \mathrm{B} \alpha$ phosphorylation and $\mathrm{NF}-\kappa \mathrm{B}$ p65 activation were 
A
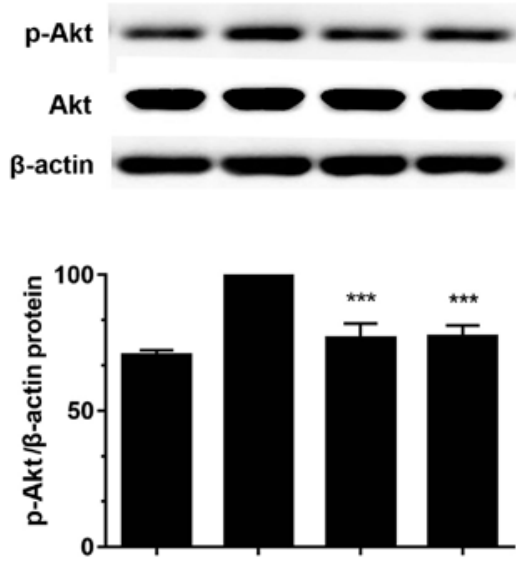

GSP $(\mu \mathrm{g} / \mathrm{ml}) \quad-\quad \quad-\quad 10 \quad 25$ LPS $(1 \mu \mathrm{g} / \mathrm{ml}) \quad-\quad+\quad+\quad+$
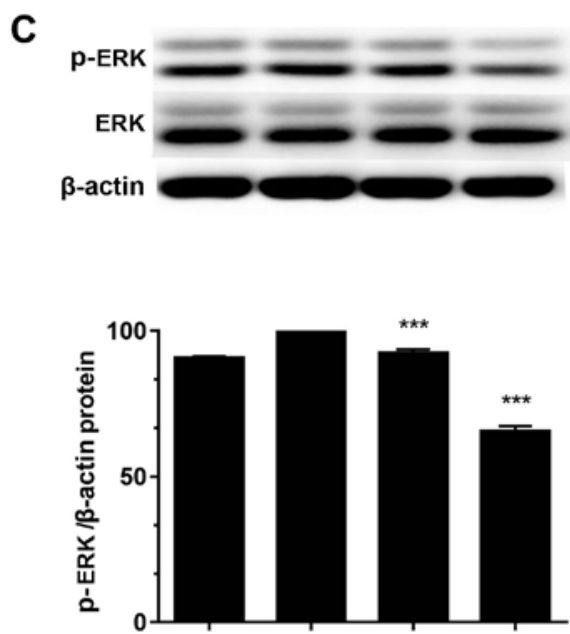

$\operatorname{GSP}(\mu \mathrm{g} / \mathrm{ml}) \quad-\quad \quad-\quad 10 \quad 25$ $\operatorname{LPS}(1 \mu \mathrm{g} / \mathrm{ml}) \quad-\quad+\quad+\quad+$
B
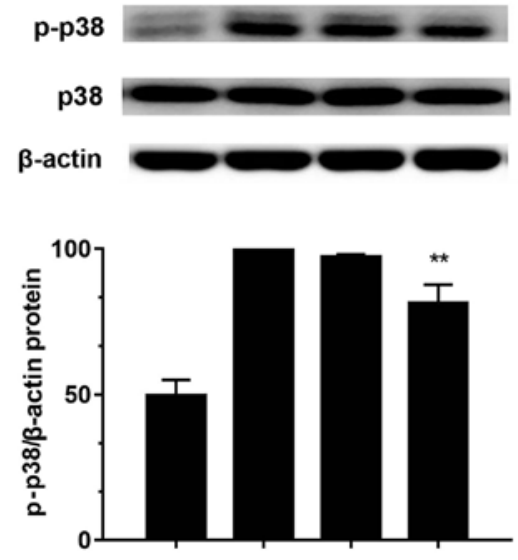

$\operatorname{GSP}(\mu \mathrm{g} / \mathrm{ml}) \quad-\quad-\quad 10 \quad 25$ LPS $(1 \mu \mathrm{g} / \mathrm{ml}) \quad-\quad+\quad+\quad+$
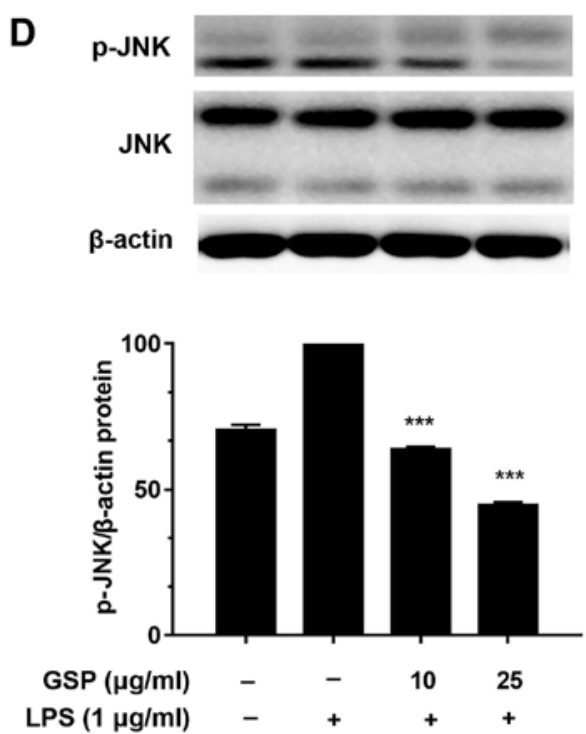

Figure 6. Effects of grape seed proanthocyanidin (GSP) on phosphorylation of MAPKs and Akt in lipopolysaccharide (LPS)-induced LX-2 cells. The expression levels of phospho-extracellular signal regulated kinase 1/2 (ERK1/2) (p-ERK), phospho-p38 (p-p38), phospho-Akt (p-Akt), and phospho-JNK1/2 (p-JNK) were analyzed by western blotting. Cells were pretreated with GSP $(10$ and $25 \mu \mathrm{g} / \mathrm{ml})$ for $24 \mathrm{~h}$ followed by LPS stimulation for $2 \mathrm{~h}$. The ratios of immunointensity between Akt or p-Akt (A), p38 or p-p38 (B), ERK or p-ERK (C), and JNK or p-JNK (D) to $\beta$-actin were calculated. Data are expressed as the mean \pm SD of three independent experiments. ${ }^{* *} \mathrm{p}<0.01$ and ${ }^{* * *} \mathrm{p}<0.001$ compared to LPS alone.

investigated. After pretreatment of LX-2 cells with GSP for $24 \mathrm{~h}$ and stimulation with LPS for $2 \mathrm{~h}$, western blotting was performed for $\mathrm{I} \kappa \mathrm{B} \alpha$ and $\mathrm{NF}-\kappa \mathrm{B}$ p65. Fig. 5 shows that the total protein expression levels of $\mathrm{I} \kappa \mathrm{B} \alpha$ and $\mathrm{NF}-\kappa \mathrm{B}$ p 65 were unaffected by treatment with LPS and pretreatment with GSP (Fig. 5A and C). However, while LPS treatment increased the protein levels of phosphorylated $\mathrm{I} \kappa \mathrm{B} \alpha$ and $\mathrm{NF}-\kappa \mathrm{B}$ p 65 , pretreatment with GSP significantly decreased levels of both these proteins (Fig. 5B and D).

Effects of GSP on phosphorylation of Akt and MAPKs in LPS-induced LX-2 cells. To investigate whether inhibition of the inflammatory response by GSP occurs via the PI3K/Akt and MAPK pathways, we studied the effects of GSP on LPS-stimulated phosphorylation of upstream kinases such as Akt, ERK, p38, and JNK in LX-2 cells. Total protein was extracted $2 \mathrm{~h}$ after LPS stimulation, and the expression of ERK, Akt, p38, JNK, and their phosphorylated proteins was examined. The treatment of LX-2 cells with LPS induced increases in expression levels of phosphorylated Akt, ERK, p38 and JNK (Fig. 6). However, while the protein level of each kinase stayed constant, the expression level decreased with pre-incubation with GSP. These results suggest that GSP pretreatment interferes with key signaling pathways, including Akt and p38 MAPK. These results indicate that activation of pro-inflammatory mediators is suppressed by GSP pretreatment of HSCs.

Effects of GSP on IL-8 cytokine production in LPS-induced $L X-2$ cells. To further analyze the anti-inflammatory effects of GSP, we used ELISA to measure the production of IL- 8 in LPS-stimulated LX-2 cells. The expression level of IL-8 was markedly increased after LPS treatment, but was significantly decreased in the GSP-treated cells (Fig. 7). The inhibitory 


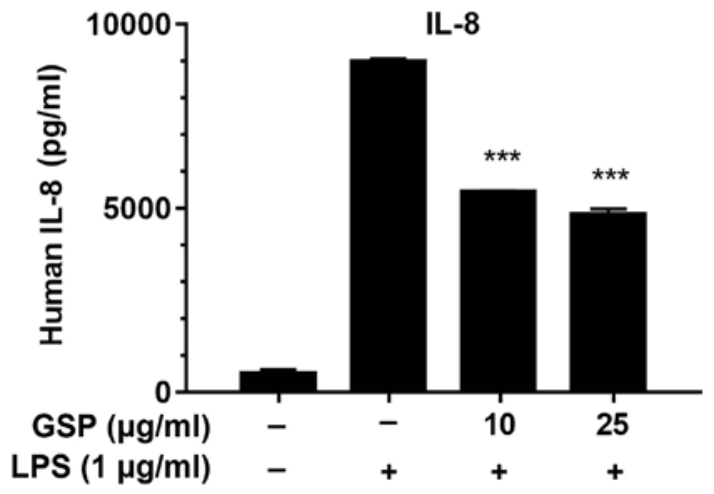

Figure 7.Effects of grape seed proanthocyanidin (GSP) on interleukin-8(IL-8) cytokine production in lipopolysaccharide (LPS)-induced LX-2 cells. Cells were pretreated with GSP (10 and $25 \mu \mathrm{g} / \mathrm{ml})$ for $4 \mathrm{~h}$ and stimulated with LPS $(1 \mu \mathrm{g} / \mathrm{ml})$ for $24 \mathrm{~h}$. The supernatants were collected and assayed by enzymelinked immunosorbent assay (ELISA). Data are expressed as the mean $\pm \mathrm{SD}$ of three independent experiments. ${ }^{* * *} \mathrm{p}<0.001$ compared to LPS alone.

effects of GSP on IL-8 protein production was correlated with the suppression of IL-8 mRNA expression (Fig. 3C). The results above indicate that the anti-inflammatory effects of GSP occur via suppression of pro-inflammatory genes or proteins related to the MAPK, Akt and NF-kB signaling pathways in LPS-stimulated cells.

\section{Discussion}

Inflammation is heavily involved in liver fibrosis. HSCs play an active role in this process via interactions with diverse types of immune cells (14). Inflammation may often coexist with liver fibrosis after liver insult. During inflammatory progression, lymphocytes and neutrophils may invade the liver and have direct effects on HSCs. The immune cells are affected by activated HSCs, which secrete chemokines and cytokines such as TGF- $\beta$, IL-10 and IL-6 (23). Medicinal plants reduce liver fibrosis and inflammation by downregulating hepatic expression and secretion of inflammatory cytokines. Inflammatory cytokines targeted by plant-derived compounds include TNF- $\alpha$, IL-1 $\alpha$, IL-1 $\beta$, IL-2, IL-4, IL-6, IL-12, IL-18 and IFN- $\gamma$. Pro-inflammatory cytokines can regulate the synthesis of a wide variety of acute phase proteins in the liver. They are also involved in the pathogenesis of liver cirrhosis and fibrosis (24). It is possible that during the inflammatory response, chemoattraction of various immune cells is promoted by human HSCs. That is, activated HSCs can evoke neutrophil chemotaxis after

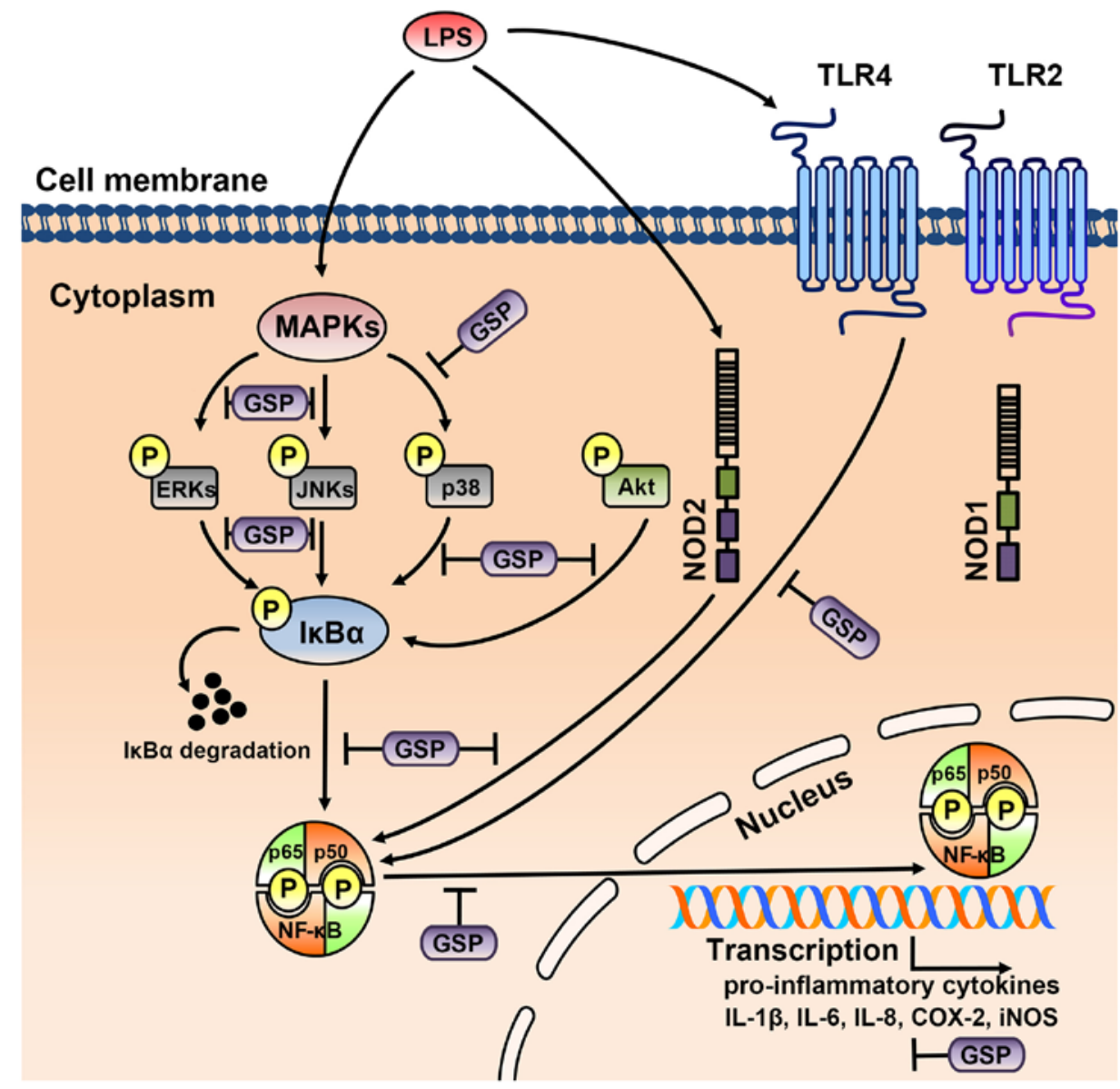

Figure 8. Schematic diagram illustrating the signaling pathways involved in the anti-inflammatory effects of grape seed proanthocyanidin extract in lipopolysaccharide (LPS)-induced hepatic stellate cells (HSCs). Grape seed proanthocyanidin (GSP) inhibited LPS-induced nuclear factor- $\kappa \mathrm{B}$ (NF- $\kappa \mathrm{B})$ activation as well as IкB $\alpha$ phosphorylation. GSP suppressed activation of three MAPKs (ERK, JNK and p38) and Akt, leading to reduced levels of Toll-like receptor 4 (TLR4), NOD2, cyclooxygenase-2 (COX-2), and inducible nitric oxide synthase (iNOS) in LPS-induced LX-2 human HSCs. 
secreting IL-8, a neutrophil chemoattractant, and macrophage inhibitory protein-2 (MIP-2) $(25,26)$. Accordingly, both the inflammatory response and liver fibrosis are affected by HSCs. Previous studies indicate that HSCs are a major factor in liver fibrosis and hepatic inflammation. Thus, inhibition of the secretion of pro-inflammatory cytokines by HSCs is an important strategic element for inhibiting inflammation and fibrosis of the liver.

Our results demonstrated that GSP noticeably suppressed the expression of IL-1 $\beta$, IL-6 and IL-8 (Fig. 3). Inflammatory stimuli (such as LPS treatment in HSCs) induce excessive production of cytokines, which intensifies the immune response and subsequent inflammation (27). Therefore, anti-inflammatory therapies often target pro-inflammatory cytokines, supporting our findings that GSP has anti-inflammatory activity via inhibition of the protein expression of IL-1 $\beta$, IL-6 and IL-8 (Figs. 3 and 7).

TLRs, particularly TLR2 and TLR4, are central mediators of inflammation during liver fibrosis. LPS signals are transmitted via TLR2 and TLR4 to the intracellular compartment, and thus, the two transmembrane receptors play important roles in the immune system (28). In this study, we demonstrated that GSP regulates TLR4 gene transcription in activated human HSCs (Fig. 2B). GSP could be employed for the inhibition of LPS/TLR4 signaling to prevent liver fibrosis. These results also suggest that GSP plays a regulatory role in inflammation at the level of TLR transcription. NODs and TLRs are a central part of the mammalian innate immune response (29). For example, NOD1 and NOD2 are primarily involved in mediating antibacterial defenses (30). We found that GSP downregulated LPS-induced NOD2 expression (Fig. 2D). These results suggest that both ligands for NOD2 and TLR4 may synergistically improve innate immune responses for the induction of the inflammatory response in HSCs (Fig. 2B and D).

iNOS and COX-2 play critical roles in the pathogenesis of certain types of human cancer and inflammatory disorders (31). Therefore, anti-inflammatory agents that inhibit iNOS and COX-2 genes may be used as potential therapeutics to treat inflammatory and infectious diseases. The results of this study indicate that GSP can efficiently inhibit the expression of iNOS and COX-2 (Fig. 4). NF- $\kappa$ B, a transcription factor, plays a role in the regulation of the expression of inflammatory mediators such as IL-1 $\beta$, IL-6, iNOS and COX-2 (22). When activated by stimuli such as LPS, $\mathrm{NF}-\kappa \mathrm{B}$ dissociates from $\mathrm{I} \kappa \mathrm{B} \alpha$ to become an active form, and I $\mathrm{B} \alpha$ is degraded. In this study, we found that GSP attenuated LPS-stimulated phosphorylation of NF- $\kappa \mathrm{B}$ in HSCs by blocking $\mathrm{I} \kappa \mathrm{B} \alpha$ phosphorylation. The MAPK pathway is involved in the expression and regulation of inflammatory mediators including iNOS and COX-2, as well as in the activation of NF- $\mathrm{B}$ (32). Since GSP inhibits $\mathrm{NF}-\kappa \mathrm{B}$ activation, we proposed the hypothesis that the MAPK pathway is involved in the attenuation of inflammatory mediators. This study showed that GSP decreased LPS-stimulated activation of MAPK in HSCs (Figs. 5 and 6). We believe that GSP exerts its anti-inflammatory effects partly by the attenuation of MAPK and NF- $\kappa \mathrm{B}$ activation.

LPS is a strong activator of the PI3K/Akt and MAPK pathways. It was recently shown that the PI3K/Akt pathway plays an important role in the regulation of LPS-induced acute inflammatory responses (33). However, its role in the regulation of $\mathrm{NF}-\kappa \mathrm{B}$ transactivation is still unclear. In this study, LPS-stimulated phosphorylation of p38 MAPK and Akt was suppressed following GSP treatment. This suggests that the suppression of LPS-induced secretion of pro-inflammatory cytokines in HSCs may be correlated with the suppression of p38 MAPK phosphorylation by GSP (Fig. 6). Our current findings suggest that GSP may block LPS-induced NF- $\kappa \mathrm{B}$ activation by inhibiting the phosphorylation of MAPKs and Akt, subsequently decreasing the protein levels of iNOS, COX-2 and other related inflammatory cytokines. In addition, our results also suggest that innate immune responses may be initiated by TLR4 ligands and further increased by NOD2 ligands, resulting in the stimulation of an inflammatory response in human HSCs (Fig. 8).

In conclusion, the results of this study demonstrated that GSP suppresses many inflammatory events, including proinflammatory cytokine secretion in LPS-stimulated HSCs. That is, GSP plays a critical role in suppressing the expression of COX-2 and iNOS, as well as the expression of pro-inflammatory cytokines such as IL-8. These inhibitory effects appear to occur via inhibition of $\mathrm{I} \kappa \mathrm{B} \alpha$ phosphorylation and subsequent suppression of NF- $\kappa \mathrm{B}$ activation, p38 MAP kinase, and Akt signaling. Hence, GSP may be a potential anti-inflammatory agent with which to treat liver disease.

\section{Acknowledgements}

The present study was financially supported by a grant (B110053) from the Korean Health Technology R\&D Project, Ministry of Health and Welfare, Republic of Korea. This study was also supported by a Korea University Grant.

\section{References}

1. Bagchi D, Bagchi M, Stohs SJ, Das DK, Ray SD, Kuszynski CA, Joshi SS and Pruess HG: Free radicals and grape seed proanthocyanidin extract: Importance in human health and disease prevention. Toxicology 148: 187-197, 2000.

2. Murphy KJ, Chronopoulos AK, Singh I, Francis MA, Moriarty H, Pike MJ, Turner AH, Mann NJ and Sinclair AJ: Dietary flavanols and procyanidin oligomers from cocoa (Theobroma cacao) inhibit platelet function. Am J Clin Nutr 77: 1466-1473, 2003

3. Steinberg FM, Bearden MM and Keen CL: Cocoa and chocolate flavonoids: Implications for cardiovascular health. J Am Diet Assoc 103: 215-223, 2003.

4. Maffei Facinó R, Carini M, Aldini G, Berti F, Rossoni G, Bombardelli E and Morazzoni P: Procyanidines from Vitis vinifera seeds protect rabbit heart from ischemia/reperfusion injury: Antioxidant intervention and/or iron and copper sequestering ability. Planta Med 62: 495-502, 1996.

5. De Bruyne T, Pieters L, Witvrouw M, De Clercq E, Vanden Berghe D and Vlietinck AJ: Biological evaluation of proanthocyanidin dimers and related polyphenols. J Nat Prod 62: 954-958, 1999.

6. Ye X, Krohn RL, Liu W, Joshi SS, Kuszynski CA, McGinn TR, Bagchi M, Preuss HG, Stohs SJ and Bagchi D: The cytotoxic effects of a novel IH636 grape seed proanthocyanidin extract on cultured human cancer cells. Mol Cell Biochem 196: 99-108, 1999.

7. Li WG, Zhang XY, Wu YJ and Tian X: Anti-inflammatory effect and mechanism of proanthocyanidins from grape seeds. Acta Pharmacol Sin 22: 1117-1120, 2001.

8. Ray S, Bagchi D, Lim PM, Bagchi M, Gross SM, Kothari SC, Preuss HG and Stohs SJ: Acute and long-term safety evaluation of a novel IH636 grape seed proanthocyanidin extract. Res Commun Mol Pathol Pharmacol 109: 165-197, 2001.

9. Seki E and Schwabe RF: Hepatic inflammation and fibrosis: Functional links and key pathways. Hepatology 61: 1066-1079, 2015.

10. Iredale JP: Hepatic stellate cell behavior during resolution of liver injury. Semin Liver Dis 21: 427-436, 2001. 
11. Wang BB, Cheng JY, Gao HH, Zhang Y, Chen ZN and Bian $\mathrm{H}$ : Hepatic stellate cells in inflammation-fibrosis-carcinoma axis. Anat Rec (Hoboken) 293: 1492-1496, 2010.

12. Xu L, Hui AY, Albanis E, Arthur MJ, O'Byrne SM, Blaner WS, Mukherjee P, Friedman SL and Eng FJ: Human hepatic stellate cell lines, LX-1 and LX-2: New tools for analysis of hepatic fibrosis. Gut 54: 142-151, 2005.

13. Henderson NC and Iredale JP: Liver fibrosis: Cellular mechanisms of progression and resolution. Clin Sci (Lond) 112: 265-280, 2007.

14. Yi HS and Jeong WI: Interaction of hepatic stellate cells with diverse types of immune cells: Foe or friend? J Gastroenterol Hepatol 28 (Suppl 1): 99-104, 2013.

15. Fallowfield JA: Therapeutic targets in liver fibrosis. Am J Physiol Gastrointest Liver Physiol 300: G709-G715, 2011.

16. Duval F, Moreno-Cuevas JE, González-Garza MT, MaldonadoBernal C and Cruz-Vega DE: Liver fibrosis and mechanisms of the protective action of medicinal plants targeting inflammation and the immune response. Int J Inflamm 2015: 943497, 2015.

17. Moon JE, Kim DM and Kim JY: Anti-inflammatory effect of Rhus verniciflua stokes extract in the murine macrophage cell line, Raw264.7. J Korean Soc Appl Biol Chem 58: 481-486, 2015.

18. Piccinini AM and Midwood KS: DAMPening inflammation by modulating TLR signaling. Mediators Inflamm 21: 2010, 2010.

19. Gabetta B, Fuzzati N, Griffini A, Lolla E, Pace R, Ruffilli T and Peterlongo F: Characterization of proanthocyanidins from grape seeds. Fitoterapia 71: 162-175, 2000.

20. Mosmann T: Rapid colorimetric assay for cellular growth and survival: Application to proliferation and cytotoxicity assays. J Immunol Methods 65: 55-63, 1983.

21. Seki E, De Minicis S, Osterreicher CH, Kluwe J, Osawa Y, Brenner DA and Schwabe RF: TLR4 enhances TGF-beta signaling and hepatic fibrosis. Nat Med 13: 1324-1332, 2007.

22. Hambleton J, Weinstein SL, Lem L and DeFranco AL: Activation of c-Jun N-terminal kinase in bacterial lipopolysaccharide-stimulated macrophages. Proc Natl Acad Sci USA 93: 2774-2778, 1996.

23. Hellerbrand C, Wang SC, Tsukamoto H, Brenner DA and Rippe RA: Expression of intracellular adhesion molecule 1 by activated hepatic stellate cells. Hepatology 24: 670-676, 1996.

24. Kayano K and Okita K: Does IL-6 regulate liver fibrosis/cirrhosis directly and indirectly? J Gastroenterol 35: 250-251, 2000.
25. Czaja MJ, Geerts A, Xu J, Schmiedeberg P and Ju Y: Monocyte chemoattractant protein 1 (MCP-1) expression occurs in toxic rat liver injury and human liver disease. J Leukoc Biol 55: 120-126, 1994.

26. Maher JJ, Lozier JS and Scott MK: Rat hepatic stellate cells produce cytokine-induced neutrophil chemoattractant in culture and in vivo. Am J Physiol 275: G847-G853, 1998.

27. Reitamo S, Remitz A, Tamai K and Uitto J: Interleukin-10 modulates type I collagen and matrix metalloprotease gene expression in cultured human skin fibroblasts. J Clin Invest 94: 2489-2492, 1994.

28. Underhill DM and Ozinsky A: Toll-like receptors: Key mediators of microbe detection. Curr Opin Immunol 14: 103-110, 2002.

29. Inohara N and Nuñez G: NODs: Intracellular proteins involved in inflammation and apoptosis. Nat Rev Immunol 3: 371-382, 2003.

30. Lee MS and Kim YJ: Signaling pathways downstream of patternrecognition receptors and their cross talk. Annu Rev Biochem 76: 447-480, 2007

31. Surh YJ, Chun KS, Cha HH, Han SS, Keum YS, Park KK and Lee SS: Molecular mechanisms underlying chemopreventive activities of anti-inflammatory phytochemicals: Down-regulation of COX-2 and iNOS through suppression of NF-kappa B activation. Mutat Res 480-481: 243-268, 2001.

32. Uto T, Suangkaew N, Morinaga O, Kariyazono H, Oiso S and Shoyama Y: Eriobotryae folium extract suppresses LPS-induced iNOS and COX-2 expression by inhibition of NF-kappaB and MAPK activation in murine macrophages. Am J Chin Med 38: 985-994, 2010.

33. Schabbauer G, Tencati M,Pedersen B,PawlinskiR and Mackman N: PI3K-Akt pathway suppresses coagulation and inflammation in endotoxemic mice. Arterioscler Thromb Vasc Biol 24: 1963-1969, 2004. 